\title{
Una propuesta de sistematización para la gestión de la documentación e información arqueológica (SIA)
}

Pablo del Fresno Bernal ${ }^{\mathbf{a}}$, Alfred Mauri Martí

${ }^{\text {a }}$ Sistemes de Gestió de Patrimoni SCCL, C. Murillo, 17, 08004 Barcelona. pdfsgp@gmail.com, bentre d’Estudis Martorellencs, Pl. de la Vila, 41 - bajos, 08760 Martorell. alfred.mauri@uab.cat.

\begin{abstract}
Resumen
Presentamos aquí una propuesta para la gestión de la Información y la Documentación Arqueológica producida en el marco de una intervención arqueológica, basada en la experiencia de su aplicación en diversas intervenciones y por parte de distintos equipos de investigación.

Para ello, recurrimos a la secuencia normalizada de los procesos de gestión informativa desde el momento inmediatamente anterior al inicio de la actividad arqueológica, hasta la presentación de la documentación que muestra y demuestra la realización de la misma y los resultados obtenidos, a la vez que se crea y gestiona el archivo de la información y documentación generadas.

Así pues, consideramos la gestión de la información y de la documentación que la fija y transmite, como el pilar básico de toda actividad científica, y la preservación del conocimiento, como el objetivo definitorio de todo Sistema de Información. La propuesta de Sistema de Información Arqueológica (SIA) se concreta en tres herramientas que son a su vez las tres líneas de desarrollo actuales del proyecto:
\end{abstract}

- SgdArq: Sistema de Gestión de la Documentación Arqueológica

- SigArq: Sistema de Información Geo-Espacial Arqueológico

- PdArq: Pauta de Descripción Arqueológica

Palabras clave: arqueología, gestión, información, documentación, normalización.

\begin{abstract}
The aim of this paper is to introduce a new proposal for Archaeological Information and Records management, in order to handle effectively those files arising from archaeological intervention. The research system is based upon our experience of practical development in different fieldwork activities developed by several research teams.

This system is explained by means of normalizing information management processes since the immediately previous moment to the beginning of archaeological activities to the final submission of reports evidencing the work carried out and showing the obtained results. Along this process, archival files arising from the archaeological record are created and stored.

Accordingly, we consider the management of information and all related files recording and transmitting these data as the underpinning pillar of every scientific activity. Hence, preservation of knowledge is the main goal of every Information System. Our proposal of Archaeological Research Information System (ARIS) is focused in the development of three tools, which currently are the main development lines of the project:
\end{abstract}

- SgdArq: Archaeological Records Management System (ARMS)

- SigArq: Archaeological Geo-Spatial Information System (ArqGIS)

- PdArq: Archaeological Descriptio Schedule (ARS)

Keywords: archaeology, management, Information, records, normalization. 


\section{Introducción}

La evolución metodológica del proceso de excavación arqueológica y del sistema de registro, así como el desarrollo del trabajo en equipo, interdisciplinar y en un entorno orientado a la Ciencia Abierta u Open Science, hacen necesario disponer de una metodología y de unos instrumentos adecuados para una gestión eficiente y segura de la información que cumpla los requisitos básicos de cualquier sistema de gestión documental y permitan asegurar la autenticidad, la integridad, la fiabilidad y la recuperación.

Estos cuatro requisitos son, a su vez, clave en lo que se refiere también al rigor científico ya que con ello se garantiza el acceso a las fuentes de información primaria que constituyen el registro arqueológico en todas sus variedades tipológicas: información descriptiva, cartográfica, gráfica, etc. Un aspecto que no siempre ha merecido suficiente atención, como lo demuestra la imposibilidad, en muchos casos, de recuperar documentación sobre intervenciones arqueológicas más allá de la que ofrecen los tradicionales diarios de campo (muchos de ellos extraviados), las memorias de excavación o, en los casos más extremos, exclusivamente por las fuentes bibliográficas.

Sin duda la propuesta de E.C. Harris en sus artículos de 1975 y 1979, desarrollados posteriormente en la publicación Principles of Archaeological Stratigraphy (Harris, 1989), supusieron un paso fundamental en los procesos de normalización tanto del trabajo de campo en arqueología como en la configuración del registro. No obstante, ello no es suficiente para el cumplimiento de los requisitos y objetivos que hemos señalado para la gestión y el uso compartido de la información y la documentación. Aspectos a los cuales hay que añadir que la intervención arqueológica, además, es una actividad regulada por la administración pública.

Presentamos aquí una propuesta para la gestión de la Información y la Documentación Arqueológica producida en el marco de una intervención arqueológica, basada en la experiencia de su aplicación en diversas intervenciones y por parte de distintos equipos de investigación, que responde a los cinco objetivos siguientes:

- Disponer de una metodología adecuada para una gestión eficiente y segura de la información que constituye el registro arqueológico, que cumpla con los requisitos básicos de cualquier sistema de gestión documental y permita asegurar su autenticidad, integridad, fiabilidad y recuperación a largo plazo.

- Definir y documentar los flujos de trabajo vinculados a la intervención arqueológica tanto de campo como de laboratorio.

- Normalizar las tipologías documentales y los instrumentos de registro y definir vocabularios controlados a fin de asegurar un registro arqueológico harmonizado.

- Potenciar y facilitar el trabajo en equipo, interdisciplinar y enfocado a la Ciencia Abierta u Open Science.

- Desarrollar recursos tecnológicos y de apoyo que faciliten alcanzar los objetivos anteriores.

Actuamos sobre la secuencia normalizada de los procesos de gestión informativa, desde el momento inmediatamente anterior al inicio de la actividad arqueológica, hasta la presentación de la documentación que muestra y demuestra la realización de la misma y los resultados obtenidos, a la vez que se crea y gestiona el archivo de la información y documentación generadas (Mauri, Travé y Del Fresno, 2018).

La gestión de la información y de la documentación que la fija y transmite constituyen el pilar básico de toda actividad científica, y la preservación del conocimiento deviene el objetivo definitorio de todo Sistema de Información. Para ello se han desarrollado tres herramientas que, en su conjunto, permiten avanzar en la respuesta a los objetivos propuestos:

- SgdArq: Sistema de Gestión de la Documentación Arqueológica

- SigArq: Sistema de Información Geo-Espacial Arqueológico

- PdArq: Pauta de Descripción Arqueológica 


\section{Las herramientas}

\subsection{SgdArq: Sistema de Gestión de la Documentación Arqueológica}

Su desarrollo se basa en los principios y métodos de la archivística y la gestión documental y se estructura a partir de un cuadro de clasificación funcional que define un primer grupo de series que identifica las funciones, un segundo en correspondencia con los procesos y, finalmente, el conjunto de series que corresponden a los procedimientos y en las que se integran las unidades documentales simples y compuestas. Su aplicación se hace extensiva a cualquier tipo de documento: textual, gráfico, cartográfico, etc. Identifica los tipos de información y tipologías documentales, define el mapa de formatos, los sistemas de descripción y clasificación, el esquema de metadatos, y los cuadros de acceso y de disposición de los documentos (Travé, Del Fresno y Mauri, 2020).

La clasificación funcional nos permite establecer una correlación directa entre las distintas fases operativas de la investigación arqueológica y la información que genera, los documentos que la registran y su clasificación, tal y como se muestra en la Fig. 1.

La primera fase operativa, la relativa al contexto productivo, se corresponde con las actuaciones de carácter administrativo, en un sentido amplio. Incluye los aspectos científico-técnicos, que constituyen propiamente un proyecto de intervención, el cumplimiento de los requisitos legales, y los propiamente administrativos internos y externos de la actuación y de la entidad u organización responsable de la ejecución.

La segunda fase, la gestión primaria, incluye las actuaciones vinculadas a la recogida de datos y su gestión para la construcción del registro arqueológico y el tratamiento de la información y los documentos que lo integran, durante la ejecución del trabajo de campo, constituyendo el bloque correspondiente a información primaria.

En consecuencia, cada Unidad Estratigráfica (UE) y unidades informativas de rango menor que de ella dependen, se registran de acuerdo con sus cuatro dimensiones, produciendo para ello documentación descriptiva, gráfica y cartográfica (ver Fig. 2). Tipologías documentales que se amplían cuando esta información primaria es explotada en la siguiente fase del ciclo informativo.

- Dimensión descriptiva. Incluye información sobre su clase (depósito, estructura o interfaz), definición normalizada para los casos más recurrentes (por ejemplo, amortización, muro, agujero...), origen natural o antrópico, e interpretación por medio de un vocabulario normalizado (por ejemplo, construcción, destrucción, uso, erosión...).

- Dimensión gráfica. A través de imágenes diferentes (aéreas, generales, de detalle...) que se almacenan e identifican a través de metadatos normalizados.

- Dimensión cartográfica. Conforme al método arqueológico, en función de la técnica empleada se obtendrá un plano exclusivo para cada UE con sus cotas, o una lista de coordenadas con los datos topográficos.

- Dimensión temporal. En los formularios de registro se consignan las relaciones temporales y físicas de la UE protagonista con todas aquellas con las que esté en contacto físico.

La generación de información primaria no finaliza con el fin de la intervención, no en vano, ésta es generada durante todo el ciclo de vida del proyecto científico. Las diferentes UUEE posteriormente son sintetizadas, agrupadas, en unidades de información de rango mayor: las correspondientes al proceso de síntesis estratigráfica o significación histórica. La gestión normalizada que permite su integración con información procedente del vaciado de otras fuentes, la realizamos por medio de las Unidades Topográficas (Mauri, Travé y Del Fresno, 2012). 


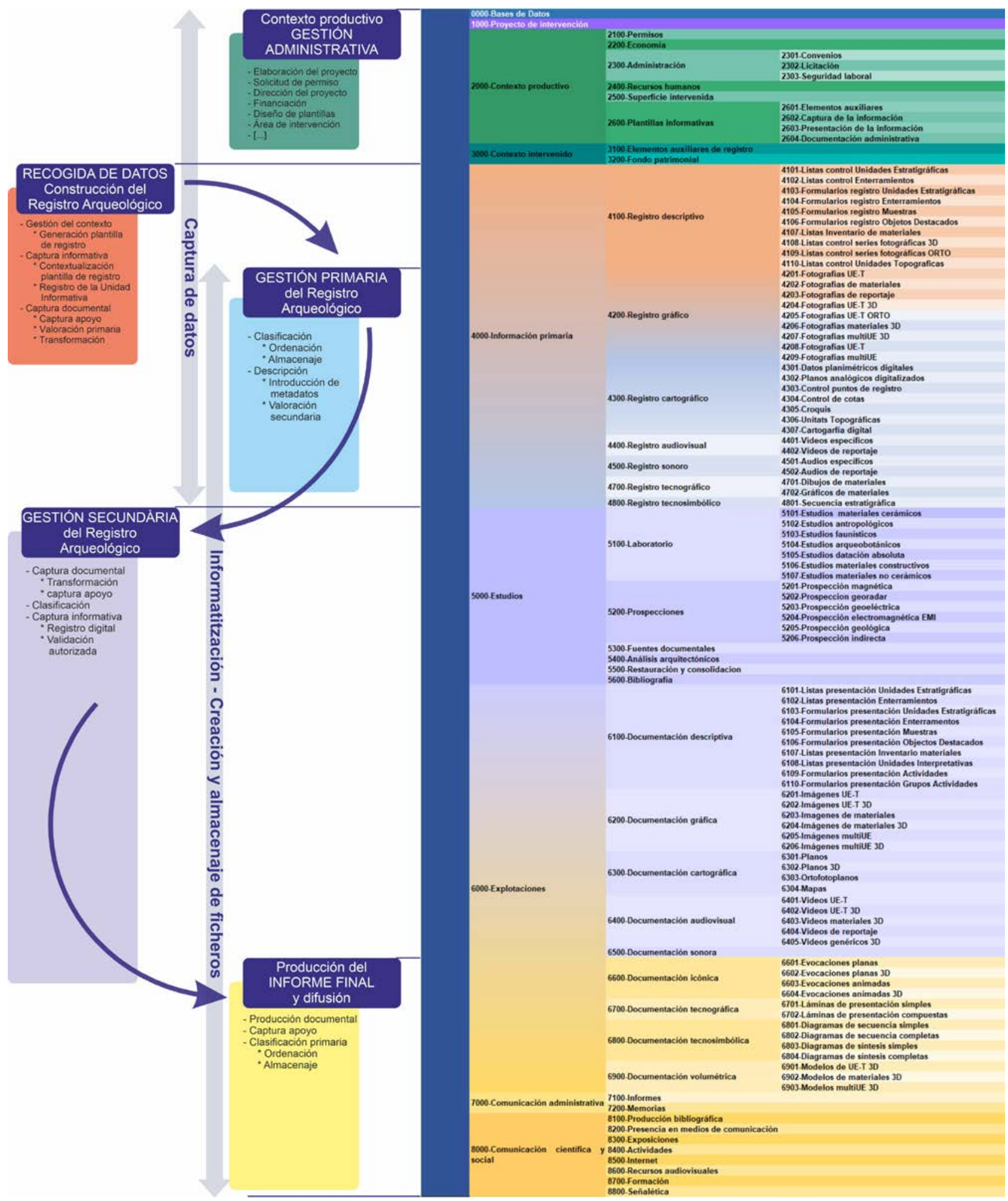

Fig. 1 Esquema general de SgdArq con indicación de la correspondencia entre las distintas fases operativas y los niveles del cuadro de clasificación (funciones, procesos y procedimientos)

En la tercera fase, que definimos como gestión secundaria, se lleva a cabo la explotación e interpretación de datos correspondientes a información y documentos derivados de la realización de estudios (análisis de laboratorio y otros, prospecciones, fuentes documentales, bibliográficas, etc.), y explotaciones a partir del registro arqueológico primario. En su conjunto constituyen la base sobre la que se desarrolla la cuarta y última fase. Ésta contempla tanto la comunicación administrativa, que responderá al ordenamiento legal y normativo al cual se encuentre sometida la 
intervención o proyecto, como la generada específicamente para ámbitos científicos y de socialización del conocimiento.

En cada una de las fases los documentos producidos se someten a un proceso de clasificación, integrándose en las series correspondientes, aplicando una pauta normalizada para su denominación en cada caso, a la vez que son descritos mediante la aplicación de metadatos, previamente definidos en el esquema específico para cada serie documental.

Si bien la clasificación constituye el esqueleto fundamental del sistema en cuanto al control de la producción y almacenaje de los documentos, la aplicación de metadatos permitirá tanto la consulta transversal a los mismos, como la aplicación de los criterios de acceso y disposición.

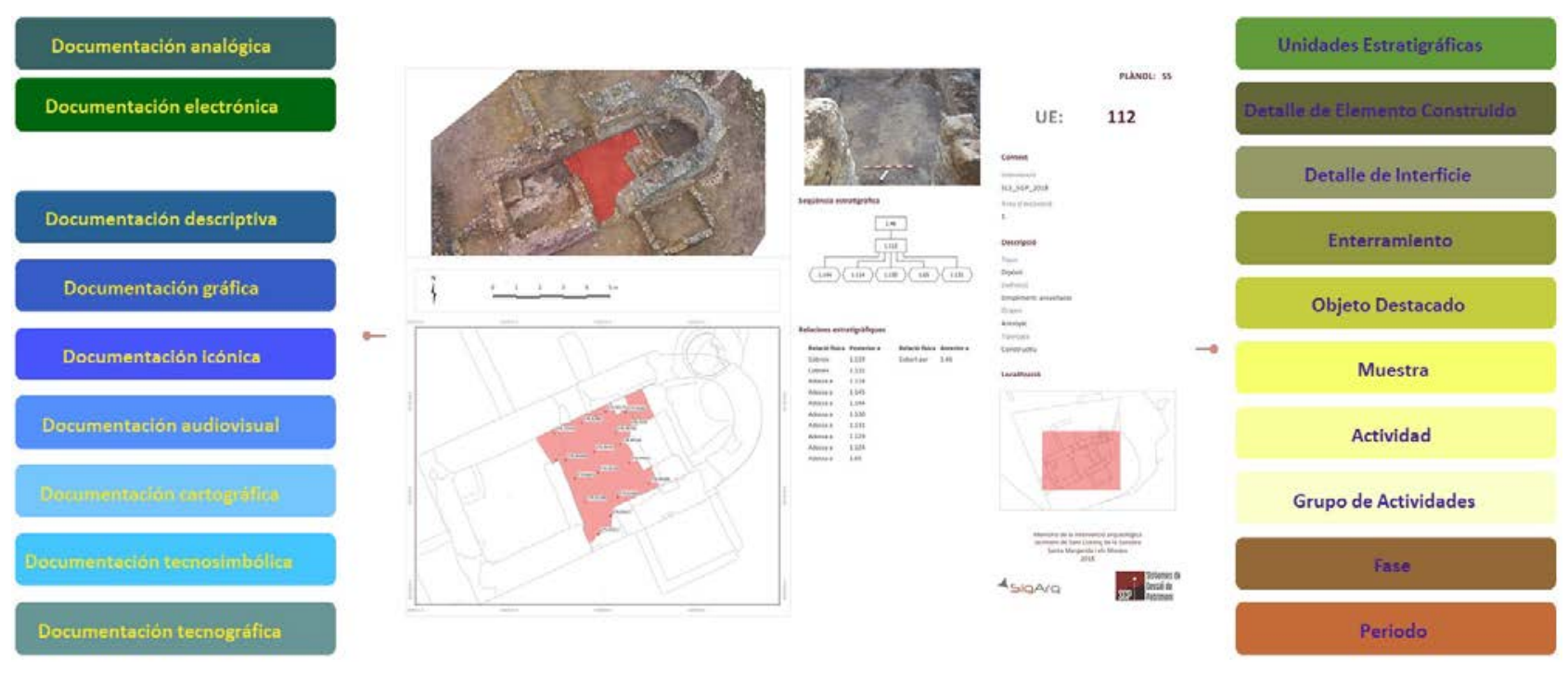

Fig. 2 Unidades de información y tipologías documentales del registro arqueológico cuya gestión es respaldada por el Sistema de Información

\subsection{SigArq: Sistema de Información Geo-Espacial Arqueológico}

SigArq presenta diferentes fases de desarrollo, la actual, la 3.0, ha sido posible por la participación de todos los agentes implicados en las anteriores versiones (SigArq 2020), especialmente la Diputación Foral de Álava, sin cuya aportación e interés hubiera sido imposible desarrollar la serie 2.0.

Sistema desarrollado por el Laboratori d’Informació Geográfica i Teledetecció (LIGIT) de la Universidad Autónoma de Barcelona y por Sistemes de Gestió de Patrimoni SCCL, con la colaboración de grupos de investigación, empresas del sector y universidades, especialmente el Centre d'Estudis Martorellencs (CEM) y Qark Arkeologia SL.

Se trata de una aplicación cliente servidor multiusuario y multilingüe en red desarrollado con software libre, que respalda la implementación normalizada de la información en un servidor centralizado. La función de SigArq dentro del SIA, es garantizar la correcta implementación informativa del registro de campo recogido según sus estándares informativos. Para ello, acude a las fuentes primarias correctamente clasificadas y descritas según la propuesta del SgdArq y genera documentación de carácter electrónico con información secundaria, es decir, aquella que es necesario consultar para poder mostrar y entender cada una de las afirmaciones que sobre la actuación arqueológica se hagan. Aquí reside el motivo central de nuestra propuesta, que otros científicos, respetando siempre los tiempos propios de la autoria intelectual y siempre sin la posibilidad de modificación o alteración, puedan acceder a la fuente primera de información de intervenciones no por ellos protagonizadas.

El usuario de forma gradual avanza a lo largo de un protocolo de informatización diseñado para reducir a la mínima expresión la posibilidad de introducir información incoherente. Por lo tanto, es un sistema que respalda la gestión normalizada de toda aquella documentación relativa al registro de las dimensiones informativas arriba descritas (Fig. 3). 


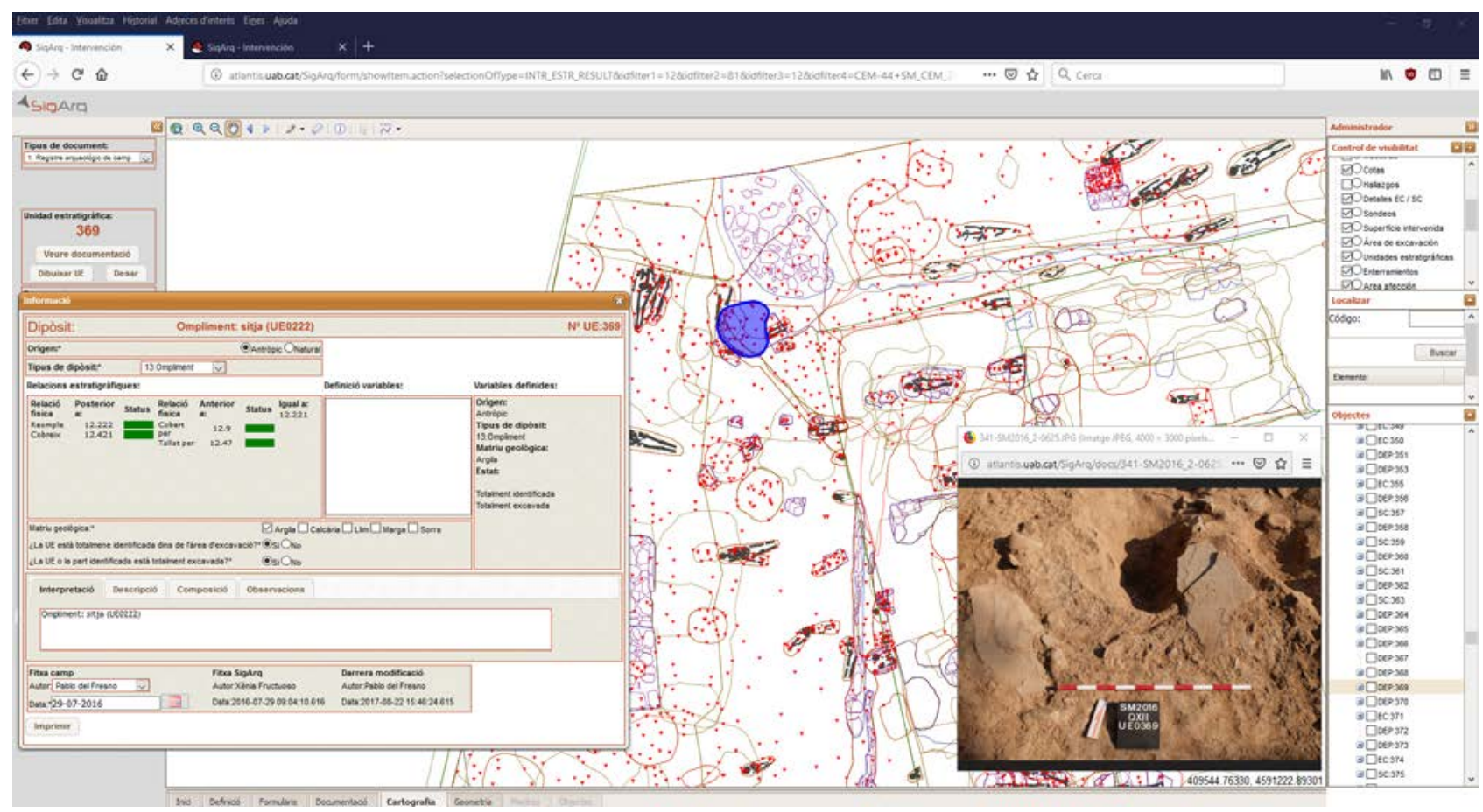

Fig. 3 Interfaz del protocolo estratigráfico correspondiente a la dimensión cartográfica

Una vez la información está almacenada y custodiada en el servidor secundario, ésta puede ser consultada y explotada por otros programas (Fig. 4), produciendo a su vez nueva documentación, por ejemplo, la plantilla de presentación de la Fig. 2, generada de forma masiva y automática para cada UE identificada en una intervención.

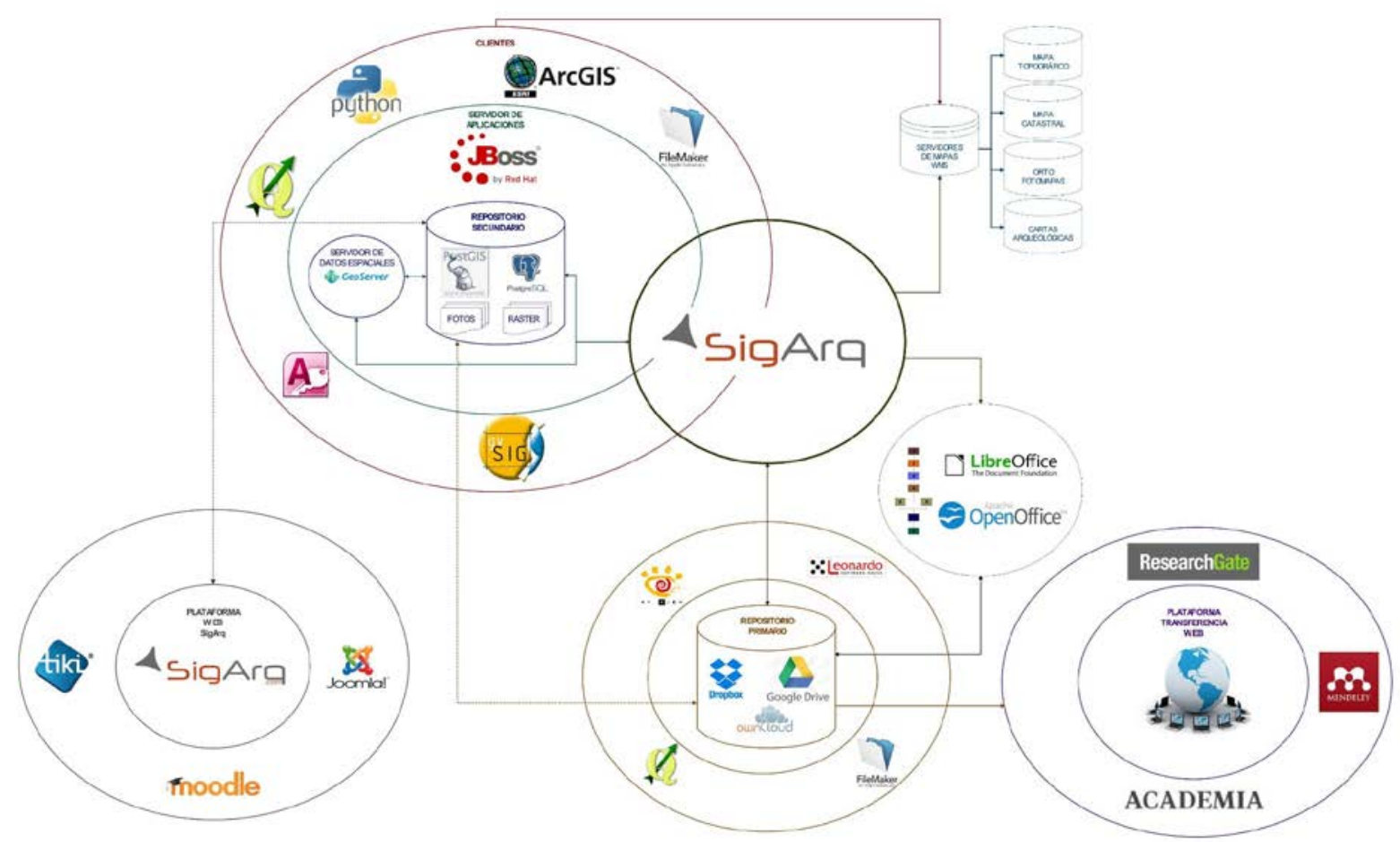

Fig. 4 Esquema conceptual general de la arquitectura de tres niveles del Sistema de Información 


\subsection{PdArq: Pauta de Descripción Arqueológica}

Ya hemos hecho referencia a la existencia de un vocabulario controlado que permite una descripción armonizada de las diferentes unidades de información. En la actualidad, este vocabulario se encuentra incrustado dentro de la aplicación SigArq. Existe por lo tanto una dependencia tecnológica para poder emplearlo. Además, su aplicación sobre casos de estudio reales, no siempre del todo satisfactoria, ha puesto de manifiesto la necesidad de replantear tanto su estructura interna como, y sobre todo, su estrategia de desarrollo.

Por todas estas razones, en la actualidad, y pendientes de poder contar con los recursos y las complicidades necesarias, nos planteamos un cambio de paradigma en lo que a la construcción de la normalización descriptiva hace referencia. En primer lugar, ir más allá del vocabulario controlado y comenzar a desarrollar una ontología, con todo lo que eso conlleva, integrando sensibilidades, casuísticas y necesidades que vengan más allá de la actual comunidad de usuarios de nuestro sistema. En segundo lugar, romper la dependencia tecnológica, es decir, respondiendo al motivo de nuestra propuesta, que se produzca registro armonizado con independencia del equipo humano, cronotipología de yacimiento y ahora, del equipo tecnológico empleado, siempre y cuando se aplique la nueva propuesta ontológica consensuada.

\section{Resultados de la experiencia}

La experiencia presentada aquí es fruto de un proceso que se inició a mediados de la década de 1990 con la incorporación de un Sistema de Información Geográfica en paralelo al sistema de bases de datos que veníamos utilizando en nuestros proyectos y a la progresiva informatización de la documentación generada.

Si bien ello representó un paso importante en la mejora de la calidad del registro arqueológico y en su gestión, también se evidenció la necesidad de avanzar hacia el diseño de un sistema de gestión integrada, que a su vez permitiera mejorar la eficiencia en la creación del registro arqueológico y en la producción de los informes y memorias. Una tarea habitualmente ardua y que no siempre es correctamente valorada en cuanto a la carga de trabajo que supone.

La concreción de la propuesta, si bien se encuentra en desarrollo y mejora constantes, se plasmó en una primera publicación (Mauri; Travé y Del Fresno, 2012) y, especialmente, con la tesis Sistema de Información Arqueológica: propuesta de normalización, desarrollo conceptual e informático (Del Fresno, 2016). El Centre d’Estudis Martorellencs, en sus proyectos de intervención en los yacimientos de Santa Margarida (Travé et al., 2019) y Sant Genís de Rocafort, en Martorell (Barcelona); Sistemes de Gestió de Patrimoni en los yacimientos del Castell-Convent de Sant Raimon de Penyafort y Sant Llorenç de la Sanabre (Del Fresno y Socorregut, 2018), en Santa Margarida i els Monjos (Barcelona); y Qark Arqueología en los yacimientos de Santa Catalina (Mansilla de la Sierra), Eras de San Martín Graccurris (Martínez, Del Fresno, y Aguirre, 2019), (Alfaro) y Contrebia Leucade (Aguilar del Río Alhama), todos ellos en La Rioja, vienen utilizando las herramientas que hemos comentado, con resultados satisfactorios y aportaciones que han permitido y han de permitir una mejora continua, imprescindible para dar respuesta a las nuevas necesidades que derivan de la evolución metodológica e instrumental de la investigación arqueológica.

Hemos observado reticencias por parte de algunos equipos para incorporar el uso del SIA propuesto, fenómeno que no es ajeno a otras propuestas de gestión digital como Piedrac, Inari Ais, Stratify, Harris Matrix Composer o Arched (en este último caso ya de difícil localización) (Díaz y Mier, 2019). Ello se debe en buena medida a la preocupación que despierta el hecho de disponer de volúmenes importantes de información y documentación ya generada que, en apariencia, parecerían perderse. Es cierto que la introducción retroactiva de esta información requiere de un esfuerzo importante, pero la realización o no de este trabajo en ningún caso compromete el correcto funcionamiento del sistema; antes al contrario, la incorporación paulatina de registros anteriores cuestiona, valida y perfecciona el propio desarrollo de la herramienta.

El objetivo principal de nuestra propuesta, en cualquier caso, busca llamar la atención a la comunidad arqueológica sobre la necesidad de trabajar con protocolos normalizados que permitan un trabajo verdaderamente interdisciplinar y en los que se puedan aunar esfuerzos realizados desde las universidades y centros de investigación, la empresa privada y los órganos de gestión pública. En este sentido, SigArq permite una generación atomatizada de informes y memorias de excavación a partir de plantillas predeterminadas que se adecuan a los criterios marcados por la administración pública. 
En su versión actual, las plantillas de edición se rigen por las pautas propuestas por la Generalitat de Catalunya, pero son adaptables a las directrices marcadas por cualquier otra administración. La herramienta como tal, se fundamenta en la normalización de protocolos y es precisamente esa normalización la que permite un diálogo fluido con otras herramientas definidas hasta el momento para la gestión de los fondos museográficos, por ejemplo, o la elaboración de cartas arqueológicas. El encaje en la gestión pública de propuestas de este tipo pasa por garantizar una interoperabilidad de datos y de sistemas que permitan generar un entorno en donde la información pueda circular con agilidad con independencia de las herramientas concretas en uso en coyunturas determinadas.

\section{Conclusiones}

Si bien las otras experiencias de software citadas permiten la gestión del registro arqueológico con mayor o menor detalle, la propuesta que aquí se ha presentado aborda la gestión de la intervención arqueológica considerándola no solamente en sí misma, sinó en todo su contexto administrativo y científico. Por tanto, además de normalizar el registro arqueológico en un sentido estricto, se normaliza también la gestión de las intervenciones desde su planificación hasta la generación de documentos técnicos, además de la información derivada de prospecciones, estudios especializados y análisis. Es de destacar la incorporación de plantillas de edición automatizada para la generación de informes y memorias.

La aplicación del SIA ha reportado a los equipos participantes en el proyecto una clara mejora en la sistematización y calidad del registro arqueológico, a la vez que la mejora en la eficiencia en los aspectos ya comentados. Somos conscientes de las limitaciones que aun presenta la propuesta, pero hoy por hoy, representa una alternativa probada a la disparidad de métodos y sistemas de gestión de la información arqueológica que dificultan en gran medida el intercambio de información y no garantizan su preservación a largo plazo. Sin duda estos son los mayores retos a los que debemos dar solución, de modo que las distintas propuestas permitan la interoperabilidad entre ellas, evitando que la información devenga cautiva de soluciones informáticas concretas.

En realidad, uno de los elementos que refuerza el potencial del sistema, a nuestro juicio, es la trazabilidad del mismo que se fundamenta en los tres pilares -SgDArq, SiGArq, PdArq. La gestión normalizada, con independencia de la aplicación concreta con la que se lleve a cabo, es la que finalmente garantiza la interoperabilidad del conjunto y permite recuperar la información generada en cualquier momento o fase del proceso de investigación. En un escenario ideal de trabajo compartido, se gestiona documentación no sólo por y para el equipo generador de la misma, sino -y sobre todopara otros profesionales del sector. Los retos que tenemos por delante implican necesariamente un camino de cooperación frente al de la competencia.

\section{Referencias}

Del Fresno, P. (2016). Sistema de Información Arqueológica: propuesta de normalización, desarrollo conceptual e informático (Tesis doctoral). Universidad del País Vasco, Vitoria. Recuperado de https://www.academia.edu/23836202/

Del Fresno, P., y Socorregut, J. (2018). Memòria de la intervenció arqueològica preventiva a Sant Llorenç de la Senabre d'acord amb la resolució d'autorització 470 K121 N-661 2018-1/22745 Santa Margarida i els Monjos (Alt Penedès). Memoria de excavación inédita. Recuperado de

https://www.academia.edu/40432667/Mem\%C3\%B2ria_de_la_intervenci\%C3\%B3_arqueol\%C3\%B2gica_preventiva_a_Sant_L loren\%C3\%A7_de_la_Sanabra._2018

Díaz, I., y Mier, V. (2019). Softwares aplicados a la arqueología [Mensaje en un blog]. Recuperado de https://mundogis.info/software-libre/

Harris, E. C. (1989). Principles of Archaeological Stratigraphy. San Diego, USA: Academic Press Inc.

Martínez, J. M., Del Fresno, P., y Aguirre, A (2019). Intervenciones arqueológicas en Graccurris durante el año 2018. Graccurris. Revista de Estudios Alfareños, 30, 17-39. 
Mauri, A., Travé, E., y Del Fresno, P. (2012). An Integrated Implementation of Written and Material Sources - Conceptual Challenge and Technological Resources. En Ollich (Ed.), Archaeology. New Approaches in Theory and Techniques (pp. 41-64). Rijeka, Croatia: InTech.

Mauri, A., Travé, E., y Del Fresno, P. (Junio de 2018). Research Information System (RIS): General principles and applications for Information Management, Open Science \& the Humanities Conference, Barcelona 2018. Barcelona, España. Recuperado de https://www.academia.edu/37341364/

SigArq (2020). Sistema de Información Geoespacial Arqueológico. Recuperado de http://www.sigarq.es/home/

Travé, E., Navarro, R., Mauri, A., Farreny, M., Del Fresno, P., y Socorregut, J. (2019). De l’església paleocristiana a la sagrera medieval: Transformacions estructurals i ordenament intern del jaciment de Santa Margarida (Martorell, Barcelona). En López (Ed. lit.) Tarraco Biennal. Actes 4t Congrés Internacional d'Arqueologia i Món Antic. VII Reunió d'Arqueologia Cristiana Hispànica: el cristianisme en l'Antiguitat Tardana. Noves perspectives (pp. 181-188). Tarragona, España: Universitat Rovira i Virgili - Institut d’Estudis Catalans.

Travé, E., Del Fresno, P., y Mauri, A. (2020). Ontology-Mediated Historical Data Modeling: Theoretical and Practical Tools for an Integrated Construction of the Past. Information. Special issue Digital Humanities, 11182; doi: 10.3390/info11040182. 